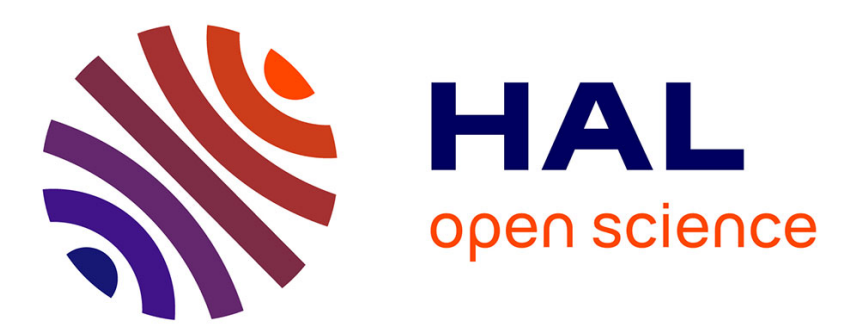

\title{
Evolution of Trophic Transmission in Parasites: Why Add Intermediate Hosts?
}

Marc Choisy, Sam P Brown, Kevin D Lafferty, Frederic Thomas

\section{To cite this version:}

Marc Choisy, Sam P Brown, Kevin D Lafferty, Frederic Thomas. Evolution of Trophic Transmission in Parasites: Why Add Intermediate Hosts?. American Naturalist, 2003, 162 (2), pp.172-181. 10.1086/375681 . hal-02502579

\section{HAL Id: hal-02502579 \\ https://hal.umontpellier.fr/hal-02502579}

Submitted on 9 Mar 2020

HAL is a multi-disciplinary open access archive for the deposit and dissemination of scientific research documents, whether they are published or not. The documents may come from teaching and research institutions in France or abroad, or from public or private research centers.
L'archive ouverte pluridisciplinaire HAL, est destinée au dépôt et à la diffusion de documents scientifiques de niveau recherche, publiés ou non, émanant des établissements d'enseignement et de recherche français ou étrangers, des laboratoires publics ou privés. 


\title{
Evolution of Trophic Transmission in Parasites: Why Add Intermediate Hosts?
}

\author{
Marc Choisy, ${ }^{1}$ Sam P. Brown, ${ }^{2}$ Kevin D. Lafferty, ${ }^{3}$ and Frédéric Thomas ${ }^{1, *}$
}

1. Centre d'Etude sur le Polymorphisme des Microorganismes, Unité Mixte de Recherche, Centre National de la Recherche Scientifique/Institut de Recherche pour le Développement, Centre IRD, 911 Avenue Agropolis, BP 64501, 34394 Montpellier Cedex 5, France;

2. Institut des Sciences de l'Evolution, Université de Montpellier II, Place Eugène Bataillon, F-34095 Montpellier Cedex 5, France; 3. U.S. Geological Survey, Western Ecological Research Center, Marine Science Institute, University of California, Santa Barbara, California 93106

Submitted March 14, 2002; Accepted December 30, 2002; Electronically published July 21, 2003

AвstraCt: Although multihost complex life cycles (CLCs) are common in several distantly related groups of parasites, their evolution remains poorly understood. In this article, we argue that under particular circumstances, adding a second host to a single-host life cycle is likely to enhance transmission (i.e., reaching the target host). For instance, in several situations, the propagules of a parasite exploiting a predator species will achieve a higher host-finding success by encysting in a prey of the target predator than by other dispersal modes. In such a case, selection should favor the transition from a singleto a two-host life cycle that includes the prey species as an intermediate host. We use an optimality model to explore this idea, and we discuss it in relation to dispersal strategies known among freeliving species, especially animal dispersal. The model found that selection favored a complex life cycle only if intermediate hosts were more abundant than definitive hosts. The selective value of a complex life cycle increased with predation rates by definitive hosts on intermediate hosts. In exploring trade-offs between transmission strategies, we found that more costly trade-offs made it more difficult to evolve a CLC while less costly trade-offs between traits could favor a mixed strategy.

Keywords: complex life cycle, dispersal, life-history strategy, parasites, predation, trophic transmission.

\footnotetext{
* Corresponding author; e-mail: fthomas@mpl.ird.fr.
}

Am. Nat. 2003. Vol. 162, pp. 172-181. (c) 2003 by The University of Chicago. 0003-0147/2003/16202-020108\$15.00. All rights reserved.
Many organisms have complex life cycles in which they possess discrete morphological stages that use different habitats. This is widely true for parasites, a large proportion of which have multihost complex life cycles (CLCs) that exploit several host species in succession (Combes 1995; Poulin 1998). While substantial effort has been devoted to describing CLCs, relatively few studies have attempted to understand why they evolved. Disentangling the mix of adaptive and contingent forces that shaped the transition from a simple life cycle (SLC) to a CLC poses a great challenge. Current hypotheses suggest that CLCs result from adjustments to historical events or accidents or where fitness benefits are gained by adding hosts to the life cycle (Smith Trail 1980; Dobson 1989; Combes 1991b; Dobson and Merenlender 1991; Lafferty 1992, 1999; Esch and Fernandez 1993; Ewald 1995; Morand 1996; see Brown et al. 2001 for a recent review). For this article, we focus on trophically transmitted parasites and acknowledge that other types of CLCs (vector transmitted, trematode cercariae, etc.) probably have different evolutionary origins.

As highlighted by Smith Trail (1980), there are at least two important ways of analyzing life cycle complexity from the observation of current CLCs, depending on whether we consider the present definitive host or the present first intermediate host as the host for the ancestral SLC. Indeed, the ecology and evolution of SLC parasites in these different hosts is likely to be different, and, consequently, the selective factors that have favored the addition of new hosts to ancestral SLCs are unlikely to be the same. For instance, Lafferty (1999) makes the classic argument that there should be selection for parasites to survive the predation of their hosts by parasitizing their host's predator and, therefore, evolving a CLC. Brown et al. (2001) use mathematical models to suggest that SLC parasites could gain by adding a predator to their life cycle because it is a simple and cheap way for finding mates. Neither the predationsurvival nor the mate-finding scenarios can, of course, explain the transition from an SLC to a CLC when the ancestral host in the SLC ends up the definitive host in a CLC. But, current knowledge suggests that both kinds of CLC exist; while trematodes most likely began as parasites 
of molluscs and added vertebrate definitive hosts (Gibson and Bray 1994; Rhode 1994), it seems likely that many nematodes that are parasitic in vertebrate guts initially had SLCs and later added intermediate hosts (Poulin 1998).

While the literature on CLCs is full of examples that illustrate spectacular adaptations that increase the probability of transmission (e.g., Combes 1991a, 1995; Combes et al. 1994; Lafferty and Morris 1996; Thomas et al. 1997), the idea that the addition of new hosts to an SLC is per se an adaptive strategy of dispersal/transmission has received little attention (but see Dobson 1989; Anderson 1992; Ewald 1995). In this article, we explore the idea that parasites with CLCs derived from SLC parasites that ancestrally exploited a predator might have evolved as a transmission strategy because, from the parasite's perspective, using a "carrier" for its propagules (an intermediate host) may, under several circumstances, increase the probability of successful transmission (i.e., reaching a new individual of the target predator). We first develop this idea using verbal arguments and then propose and analyze a model to examine the ecological determinants of an evolutionary transition from a simple to a complex life cycle. We investigate the effect of differing degrees of antagonism between SLC and CLC strategies on the likelihood of evolutionary transitions from SLC to CLC and also take into account the possible role of parasiteincreased trophic transmission.

\section{Verbal Arguments}

The propagules of any living organism must actively or passively reach a suitable habitat to survive and develop. Dispersal strategies used by plants and animals are extraordinarily various and include self-, water, wind, and animal dispersal. Numerous animals and plants use animals for dispersal, and, in most documented cases, the carrier host suffers no harm. Obvious benefits of animal dispersal are that it is energy efficient and that the carrier host often moves to specific and predictable habitats.

Transmission of infective stages to new host habitats is an essential aspect of parasitism. We can easily understand how the propagules of an ectoparasite infecting a gregarious host species will have fewer problems dispersing to new hosts than those of an endoparasite species infecting a host species living at low population density. In the latter case, we may expect stronger selection pressure on parasites for traits that increase the probability of host encounter. Particular groups such as insect parasitoids have evolved complex adult behavioral traits that enable them to locate hosts. However, few typical parasites have such adaptations for locating hosts. The use of an intermediatehost prey by parasites exploiting predators could be viewed as a particular case of animal dispersal in which a defin- itive-host predator eats the carrier host. Consider, for example, an SLC parasite that exploits a predator species and that releases its propagules into the environment. If the probability of direct transmission is lower than the probability of infecting a prey species that will be eaten by the target host, selection could favor the transition from an SLC to a CLC with the prey species as an intermediate host. In other words, when the parasite's propagules are more likely to be dispersed to a suitable host by transiting first in one of its prey than by trying to infect it directly, selection is likely to favor this indirect but more efficient route. Note, however, that this conclusion strongly depends on the form of the trade-off between SLC and CLC life-history strategies. Indeed, there is likely to be some type of negative pleiotropy between adaptations favoring SLC and CLC life-history strategies.

There are several reasons to think that transmission could increase by adding a prey host to an SLC exploiting a predator. Indeed, predators are probably more efficient at finding a prey than parasitic infective stages are at finding a target predator. In addition, given that prey populations are usually larger than predator populations, it seems that it would be more likely for a parasite to contact a prey than a predator. Finally, by encysting in a prey, infective larval stages reduce the time to which they are exposed to the adversity of the external environment (Chabaud 1954). From an evolutionary point of view, the use of trophic transmission to improve the success of dispersal is not fundamentally distinct from other types of animal dispersal since the main difference (i.e., the death of the carrier host) can be viewed just as a particular feature resulting from being an endoparasite of a predator. Intermediate situations between no harm and the death of the intermediate host may help to defend this idea. For instance, there are examples of trophically transmitted parasites that are disproportionately present in the limbs of prey species (e.g., crab's claws or lizard tail) and that are transmitted to predators via nonlethal limb autotomy (Lafferty 1999).

In classical animal dispersal, the quality of the carrier host increases with the probability that it will bring its passenger into a suitable habitat, a parameter closely related to the ecological preference of the carrier host. In our hypothesis, the quality of the intermediate host is associated with its probability of being captured by a suitable predator. In this context, the manipulation of the host phenotype in a way that increases the vulnerability of the intermediate host to a target predator can be viewed as a mechanism through which the parasite improves the quality of the intermediate host. If the costs of finding an intermediate host and manipulating it remain lower than the cost of self-dispersing, the transition from an SLC to a CLC is likely to be favored by selection. However, since 
there is no a priori benefit of increasing the host vulnerability to predators before a CLC has been selected, host manipulation per se is unlikely to be involved in the initial switch from an SLC to a CLC.

\section{The Model}

Throughout this section, we will make the assumption that the fitness of a parasite can be expressed by its basic reproductive ratio, $R_{0}$. This is a good approximation (Anderson and May 1982), even if not universally true such as in the case of superinfection (May and Nowak 1994; Nowak and May 1994). Consider the hypothetical life cycle presented in figure 1 with two hosts, one being the prey of the other. The definitive ancestral host (predator) has a density of $N_{1}$. The potential intermediate host (prey) has a density of $\mathrm{N}_{2}$. For simplicity, we will consider only parasite life-history traits and imagine that parasites are hermaphrodites. Moreover, we consider that parasite reproduction occurs only in the predator host. New parasite individuals enter the environment at a rate $\lambda$ per individual parasite present in the predator host. From there, freeliving parasites can reach a new predator host individual at a rate $\beta_{1}$ or a prey host individual at a rate $\beta_{2}$. In the latter case, the parasite may reach the definitive host through predation, with definitive hosts preying on intermediate hosts at a rate $p$ ( $p$ being a measure of the quality of the intermediate-host prey). The parameters $\mu_{1}, \mu_{2}$, and $\mu_{3}$ represent the mortality rates of the parasites in the predator host, the prey host, and the environment, respectively. We assume that mortality in the environment does not include a dead-end contact with prey individuals for a parasite with an SLC.

In the following, the SLC subscript refers to the cycle including only the predator host, and the CLC subscript refers to the cycle including both the predator and the prey hosts. The basic reproductive ratios of such cycles derive from the parameters of figure 1 . Considering an SLC alone, we have

$$
R_{\mathrm{SLC}}=\frac{\lambda \beta_{1} N_{1}}{\mu_{1}\left(\mu_{3}+\beta_{1} N_{1}\right)} .
$$

This is simply the net rate of reproduction in the predator host $\left(\lambda \beta_{1} N_{1}\right)$ multiplied by the product of the expected life spans of the parasite in the predator host $\left(1 / \mu_{1}\right)$ and the environment $\left(1 /\left[\mu_{3}+\beta_{1} N_{1}\right]\right)$. Similarly, the basic reproductive ratio of a parasite in a CLC alone is

$$
R_{\mathrm{CLC}}=\frac{\lambda p N_{1} \beta_{2} N_{2}}{\mu_{1}\left(\mu_{2}+p N_{1}\right)\left(\mu_{3}+\beta_{2} N_{2}\right)},
$$

which is the product of the net rate of reproduction in

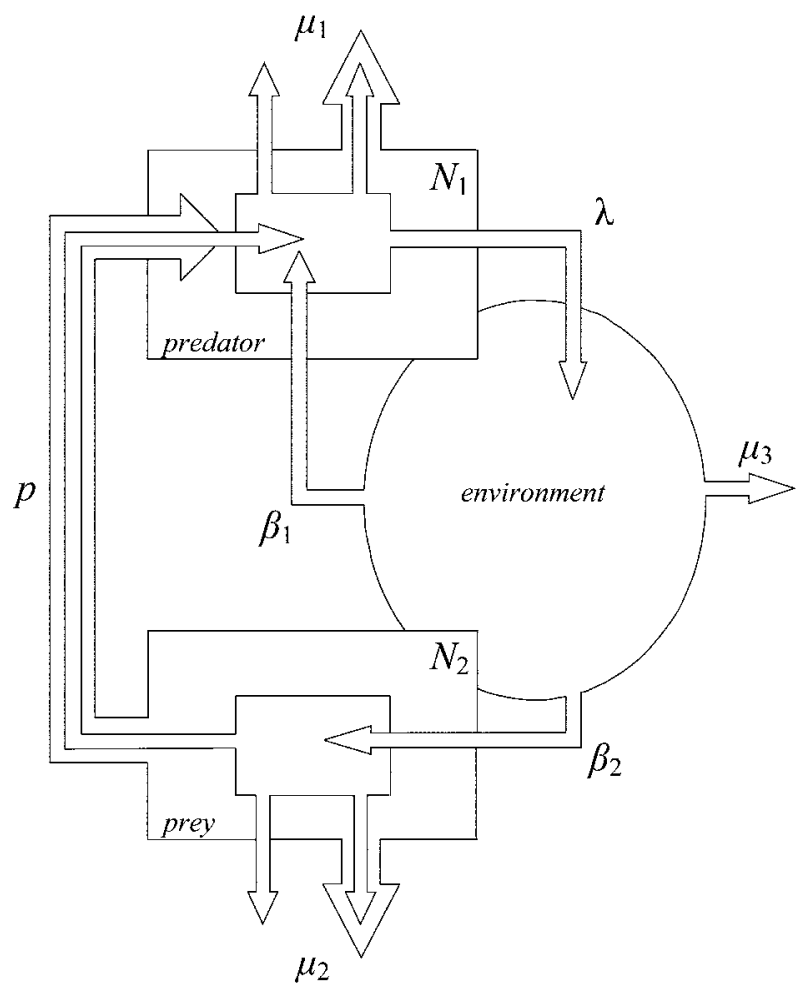

Figure 1: Flowchart of the simple life cycle and complex life cycle routes considered in our model. New parasites are released in the environment at a rate of $\lambda$ per parasite in the predator host. In an SLC, free-living parasites from the environment reach a new predator host with a transmission rate of $\beta_{1}$. In a CLC, free-living parasites from the environment reach a prey host with a transmission rate of $\beta_{2}$. Predators eat prey hosts at the rate $p$. The parameters $\mu_{1}, \mu_{2}$, and $\mu_{3}$ represent the mortality rates of the parasites in the predator host, the prey host, and the environment, respectively. In each host, we do not make a distinction between the intrinsic mortality of the parasite and parasite mortality due to host mortality. Finally, $N_{1}$ and $N_{2}$ are the densities of the predator and prey hosts, respectively.

the predator host $\left(\lambda p N_{1}\right)$ and the rate of transmission from the environment to the prey host $\left(\beta_{2} N_{2}\right)$ multiplied by the product of the expected life spans of the parasite in the predator host $\left(1 / \mu_{1}\right)$, the prey host $\left(1 /\left[\mu_{2}+p N_{1}\right]\right)$, and the environment $\left(1 /\left[\mu_{3}+\beta_{2} N_{2}\right]\right)$.

An evolutionary transition from an SLC to a CLC requires the consideration of mixed transmission strategies. Considering SLC and CLC strategies together as extremes of a continuum implies that in equations (1) and (2), the expected life span of the parasite in the environment is $1 /\left(\mu_{3}+\beta_{1} N_{1}+\beta_{2} N_{2}\right)$. With these modifications made, the expression of the basic reproductive ratio of a mixed strategy is simply the sum of $R_{\mathrm{SLC}}$ and $R_{\mathrm{CLC}}$ : 


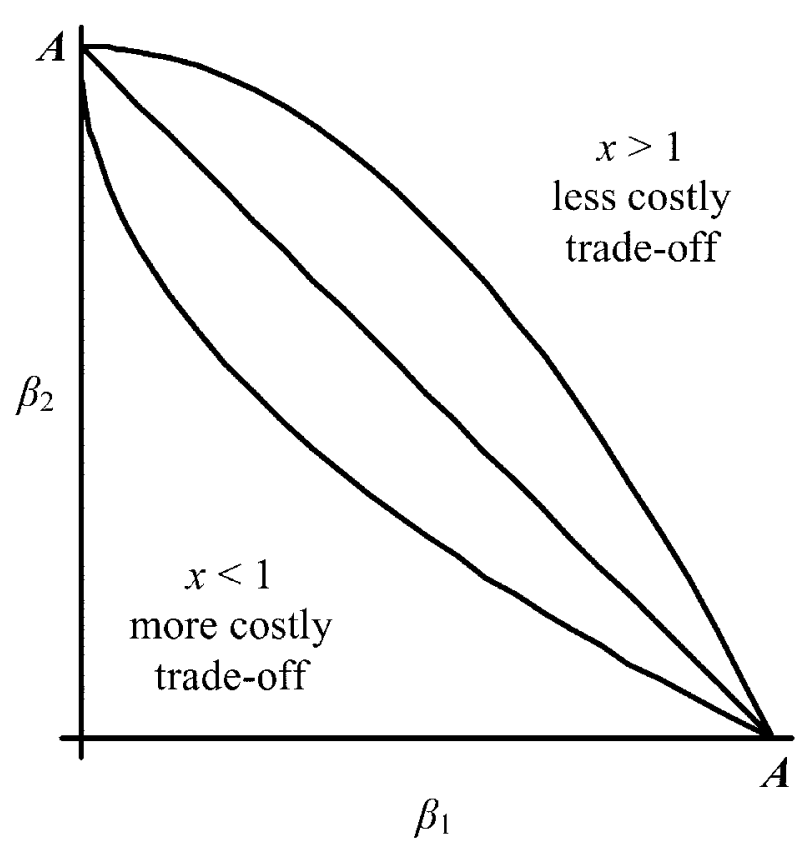

Figure 2: Trade-off between $\beta_{1}$ and $\beta_{2}$. The mathematical form of the trade-off is $\beta_{2}=A-\beta_{1}^{x}$. The straight line illustrates this trade-off for $x=1$. Below this line, $x<1$, which corresponds to a more costly tradeoff between an SLC and a CLC strategy. Above the straight line, $x>1$, which corresponds to a less costly trade-off between an SLC and a CLC strategy. Here, we have $x=0.5$ for the more costly trade-off and $x=$ 2 for the less costly trade-off.

$$
\begin{aligned}
R_{0}= & \frac{\lambda \beta_{1} N_{1}}{\mu_{1}\left(\mu_{3}+\beta_{1} N_{1}+\beta_{2} N_{2}\right)} \\
& +\frac{\lambda p N_{1} \beta_{2} N_{2}}{\mu_{1}\left(\mu_{2}+p N_{1}\right)\left(\mu_{3}+\beta_{1} N_{1}+\beta_{2} N_{2}\right)} .
\end{aligned}
$$

The first term of equation (3) represents the basic reproductive ratio via the SLC route of transmission, whereas the second term represents the basic reproductive ratio via the CLC route. At this point, maximization of basic reproductive ratio $\left(R_{0}\right)$ is achieved by maximization of the sum of both terms of equation (3).

Consider now that an organism cannot optimize two alternative evolutionary strategies. In other words, the parasite of our model cannot optimally adapt to both hosts. This leads to the definition of a trade-off between exploitation of the two possible hosts. The mathematical formalization of a trade-off often highlights a lack of biological understanding of the traits in question and ensures that any specific mathematical form is often subjective (Mackinnon and Read 1999). It is consequently important to explore how the form of the trade-off influences the results of the model. We could use the simple linear form $\beta_{2}=A-\beta_{1}$, where the transmission parameters $\beta_{1}$ and $\beta_{2}$ are both constrained between 0 and a positive constant $A$ and where any decrease in SLC transmission $\left(\beta_{1}\right)$ results in exactly the same increase in CLC transmission $\left(\beta_{2}\right)$ and vice versa (the straight line in fig. 2). Constant $A$ is the maximal transmission rate from the environment to a host, regardless of the transmission route, and we can set $A$ as large as we wish. As we will see in the analytical section, the exact value of this constant has no effect on the outcomes of the model. Concerning the form of the tradeoff between the two transmission parameters $\beta_{1}$ and $\beta_{2}$, we can account for nonlinear relationships by considering the more general expression $\beta_{2}=A-\beta_{1}^{x}$. For $x<1$ (below the straight line in fig. 2), the initial increase in CLC transmission $\left(\beta_{2}\right)$ is more costly because it does not fully compensate a decrease in SLC transmission $\left(\beta_{1}\right)$. For $x>1$ (above the straight line in fig. 2), the initial increase in CLC transmission $\left(\beta_{2}\right)$ is less costly because it more than compensates for a decrease in SLC transmission $\left(\beta_{1}\right)$. Introducing the general form of the trade-off in equation (3) leads to

$$
\begin{aligned}
R_{0}= & \frac{\lambda \beta_{1} N_{1}}{\mu_{1}\left[\mu_{3}+\beta_{1} N_{1}+\left(A-\beta_{1}^{x}\right) N_{2}\right]} \\
& +\frac{\lambda p N_{1}\left(A-\beta_{1}^{x}\right) N_{2}}{\mu_{1}\left(\mu_{2}+p N_{1}\right)\left[\mu_{3}+\beta_{1} N_{1}+\left(A-\beta_{1}^{x}\right) N_{2}\right]} .
\end{aligned}
$$

Here, the pure SLC strategy is the ancestral state $\left(\beta_{1}=\right.$ $A$ ). A value of $\beta_{1}=0$ would correspond to a pure CLC strategy, and all values of $\beta_{1}$ between 0 and $A$ would correspond to a mixture of SLC and CLC strategies.

\section{Analytical Analysis of a Simplified Model}

The general form of the model expressed by equation (4) involves exponential parameters that make the full model difficult to resolve analytically for $x \neq 1$. However, assigning the value 1 to $x$ (linear trade-off) makes the model easy to resolve analytically. In this section, we derive analytical results of a simplified version of the model of equation (4) with $x$ set to 1 , and in the following section, we explore generalization of the results to other trade-off relationships over a range of $x$ values. In the particular case where $x=1$, figure 3 illustrates the fitness $\left(R_{0}\right)$ landscape relative to the predation rate $(p)$ and the definitivehost infection rate $\left(\beta_{1}\right)$ for different combinations of intermediate-host and environmental mortalities ( $\mu_{2}$ vs. $\left.\mu_{3}\right)$ and host densities $\left(N_{1}\right.$ vs. $\left.N_{2}\right)$. In all cases, we can see that there is no adaptive peak corresponding to an evolutionary stable mixed strategy between a pure SLC and a pure CLC (i.e., intermediate values of $\beta_{1}$ ). In figure $3 A$ and $3 B$, selection favors the SLC for all values of $p$ and 

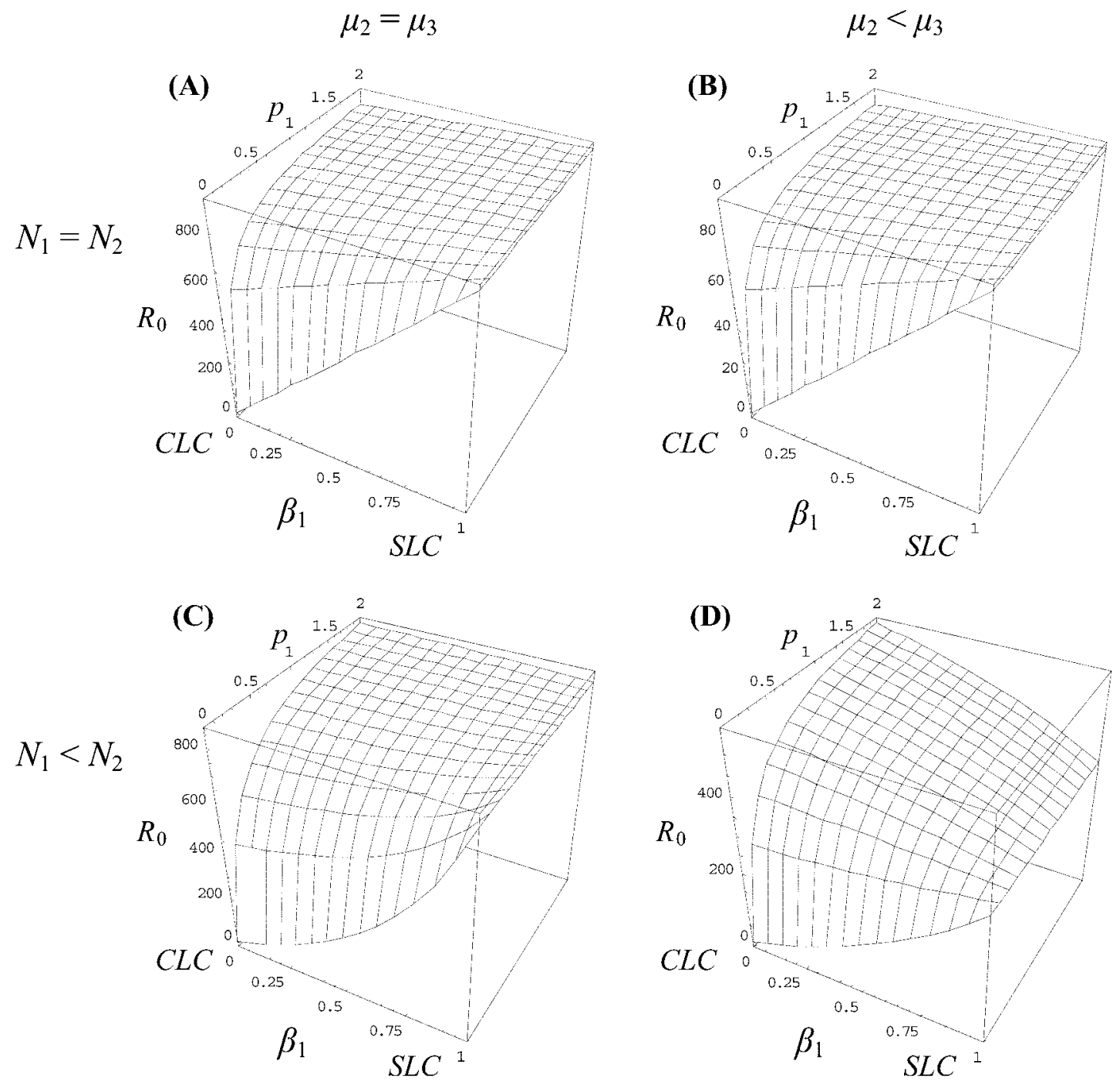

Figure 3: Basic reproductive ratio $\left(R_{0}\right)$ as a function of the ability of free-living parasites to infest a definitive host directly from the environment $\left(\beta_{1}\right)$ and the rate of predation of the definitive host on the intermediate host $(p)$. Other parameters take the following values: $N_{1}=N_{2}=0.1$ for $A$ and $B, N_{1}=0.05$ and $N_{2}=0.15$ for $C$ and $D, \mu_{3}=0.01$ for $A$ and $C$, and $\mu_{3}=0.1$ for $B$ and $D$. In all cases, $A=1, x=1, \lambda=10$, and $\mu_{1}=\mu_{2}=0.01$.

$\beta_{1}$. In figure $3 C$ and $3 D$, the adaptive strategy switches from an SLC to a CLC as the predation rate $p$ increases.

To investigate which strategy (pure SLC, pure CLC, or a mixture of SLC and CLC) selection favors, we need to consider the behavior of $R_{0}$ with respect to the parameter $\beta_{1}$. Consider the differential of $R_{0}$ with respect to $\beta_{1}$ :

$$
\frac{d R_{0}}{d \beta_{1}}=\frac{\lambda N_{1}\left[A N_{2} \mu_{2}+p\left(N_{1} \mu_{3}-N_{2} \mu_{3}\right)+\mu_{2} \mu_{3}\right]}{\mu_{1}\left(p N_{1}+\mu_{2}\right)\left[A N_{2}+\left(N_{1}-N_{2}\right) \beta_{1}+\mu_{3}\right]^{2}} .
$$

No value of $\beta_{1}$ cancels out the differential of equation (5), so selection does not favor a mixed strategy. In other words, if equation (5) is positive, selection favors a pure SLC, whereas it favors a pure CLC if equation (5) is negative. To facilitate the sign study of equation (5), consider first the differential of $R_{0}$ with respect to the predation rate $p$ :

$$
\frac{d R_{0}}{d p}=\frac{N_{1} N_{2}\left(A-\beta_{1}\right) \lambda \mu_{2}}{\mu_{1}\left(N_{1} p+\mu_{2}\right)^{2}\left[\beta_{1}\left(N_{2}-N_{1}\right)+A N_{2}+\mu_{3}\right]} .
$$

Since we showed that no mixed strategy will be favored by selection (eq. [5]), consider only the two cases of $\beta_{1}=0$ and $\beta_{1}=A$. The differential of equation (6) is 
More costly trade-off

$x<1$
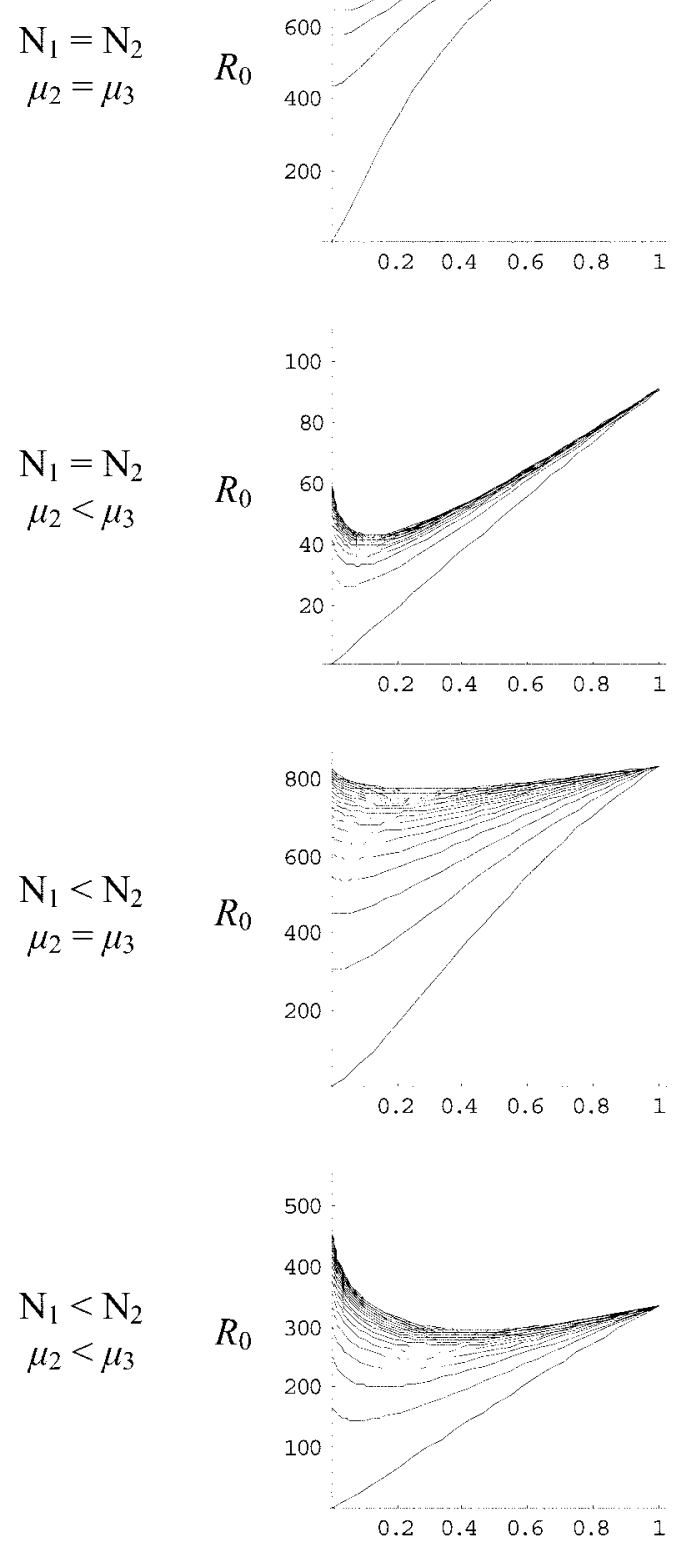

CLC $\quad \beta_{1} \quad S L C$
Linear trade-off

$x=1$

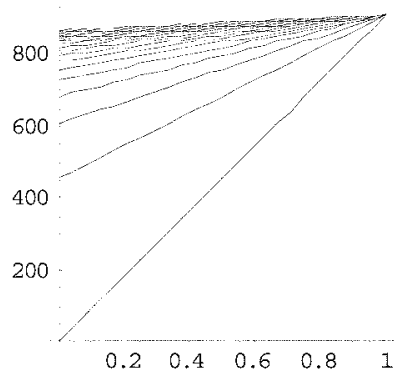

100
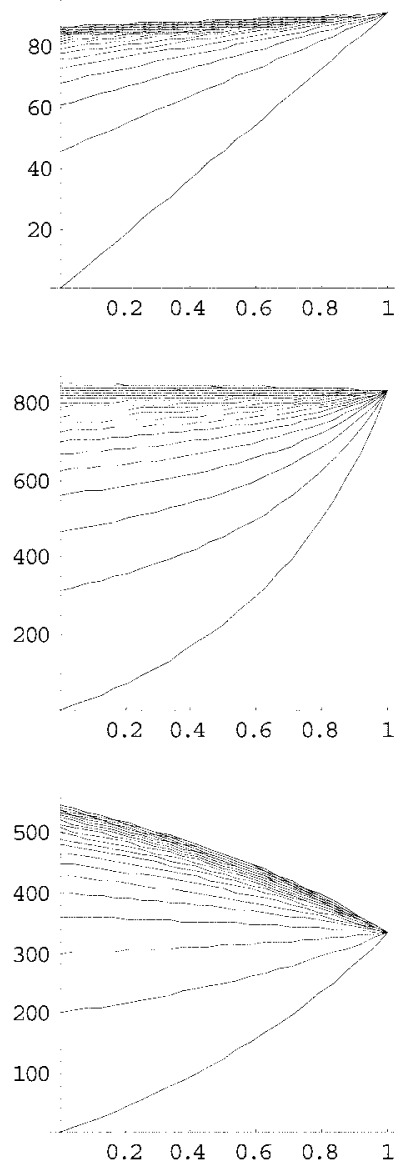

$C L C \quad \beta_{1} \quad S L C$
Less costly trade-off

$x>1$
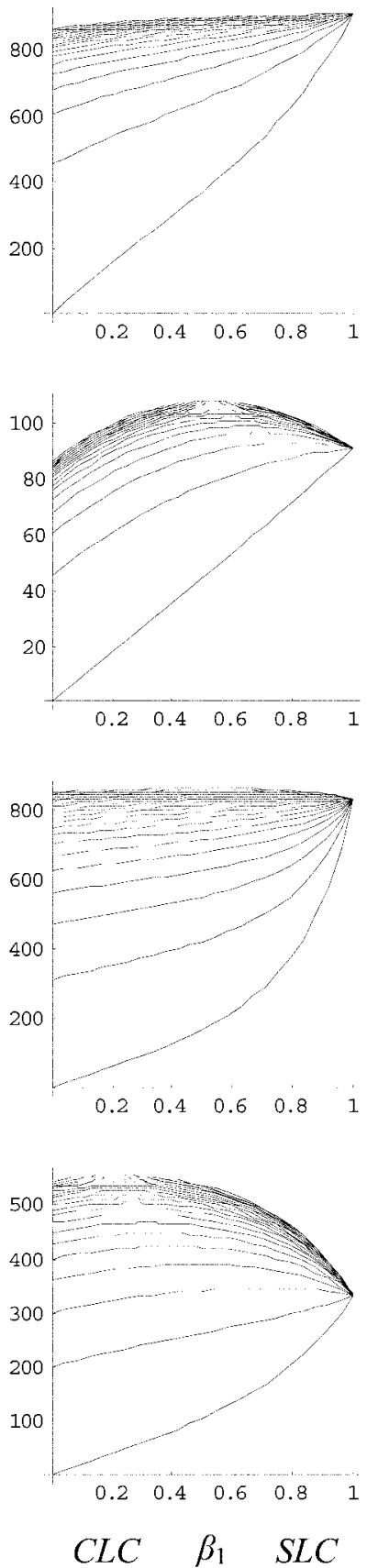

Figure 4: The influence of the trade-off form between transmission routes. Each row corresponds to the four biological cases defined in figure 3, with $x=0.2,1$, and 2 for the first, second, and third columns, respectively. The curves from bottom to top correspond with increasing values of $p$ from 0 to 2 , every 0.1 . 
More costly trade-off

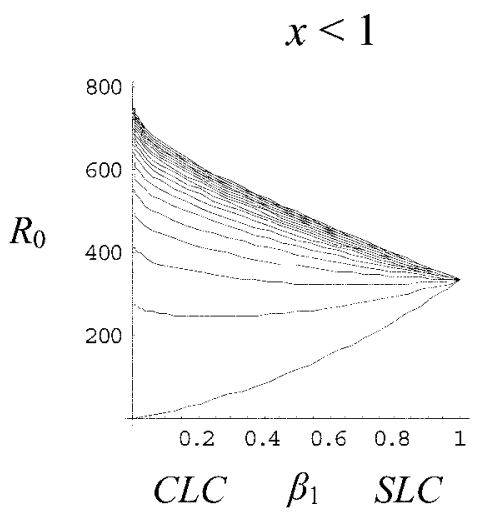

Linear trade-off

$$
x=1
$$

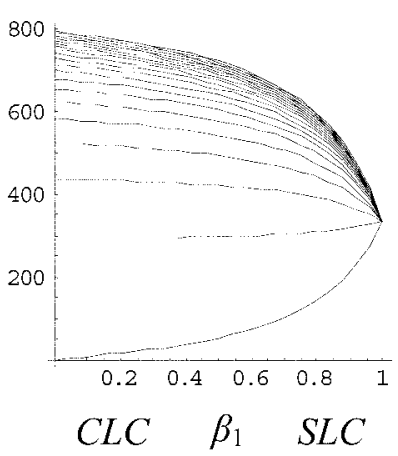

Less costly trade-off

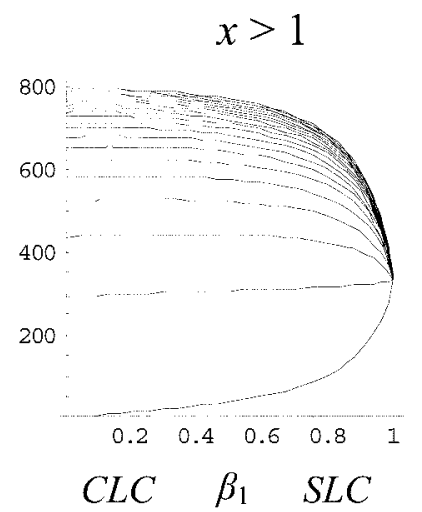

Figure 5: The influence of the ratio of predator-prey density for comparison with the three graphs of the last row of figure 4. Here, $N_{2}=0.7$ instead of $N_{2}=0.15$ in figure 4 .

strictly positive for $\beta_{1}=0$ and equals 0 for $\beta_{1}=A$. This means that if a switch from the pure SLC to the pure CLC strategy is to occur when $p$ increases, there will not be any further switchback to the pure SLC strategy when $p$ continues to increase. The consequence is that analyzing the sign of equation (5) for the two extreme values of the range of $p(0$ and its limit in $+\infty)$ is sufficient for drawing conclusions about the behavior of our model. Note that looking at $p=0$ and $p=+\infty$ does not have any particular biological significance; it is just convenient here for our mathematical analysis. If we set $p$ to 0 , equation (5) becomes

$$
\left.\frac{d R_{0}}{d \beta_{1}}\right|_{p=0}=\frac{\lambda N_{1}\left(A N_{2}+\mu_{3}\right)}{\mu_{1}\left[A N_{2}+\left(N_{1}-N_{2}\right) \beta_{1}+\mu_{3}\right]^{2}} .
$$

Because the differential of equation (7) is always positive, the basic reproductive ratio $\left(R_{0}\right)$ continuously increases with $\beta_{1}$, and selection favors a pure SLC strategy $\left(\beta_{1}=\right.$ $A$ ). This is not surprising since $p=0$ corresponds to the hypothetical case where the potential intermediate hosts are not prey of the definitive hosts at all. In this situation, a pure SLC is indeed the only alternative.

If we now let $p$ increase toward infinity, equation (5) becomes

$$
\lim _{p \rightarrow+\infty} \frac{d R_{0}}{d \beta_{1}}=\frac{\lambda \mu_{3}\left(N_{1}-N_{2}\right)}{\mu_{1}\left[A N_{2}+\left(N_{1}-N_{2}\right) \beta_{1}+\mu_{3}\right]^{2}} .
$$

Here, the sign of the differential is the same as the sign of $N_{1}-N_{2}$. If there are fewer intermediate hosts than definitive hosts $\left(N_{1}>N_{2}\right)$, then the differential of equation
(8) is positive, and we can conclude that selection will always favor the pure SLC strategy, whatever the value of the predation rate $p$. Conversely, if there are more intermediate hosts than definitive hosts $\left(N_{1}<N_{2}\right)$, then the differential of equation (8) is negative, and we can conclude that there exists a positive threshold value $p^{*}$ of the predation rate $p$ below which selection always favors a pure SLC $\left(\beta_{1}=A\right)$ strategy and above which selection always favors a pure CLC $\left(\beta_{1}=0\right)$ strategy. This threshold of $p$ is defined implicitly by

$$
\left.\frac{d R_{0}}{d \beta_{1}}\right|_{p=p^{*}}=0
$$

which yields

$$
p^{*}=\frac{\mu_{2}\left(A N_{2}+\mu_{3}\right)}{\mu_{3}\left(N_{2}-N_{1}\right)} .
$$

For the particular cases of figure 3 , we have $p^{*}=+\infty$ for figure $3 A$ and $3 B, p^{*}=1.6$ for figure $3 C$, and $p^{*}=$ 0.25 for figure $3 D$.

In summary, for selection to favor a pure CLC strategy $\left(\beta_{1}=0\right)$ requires the necessary condition that the density of intermediate hosts $\left(N_{2}\right)$ be higher than the density of definitive hosts $\left(N_{1}\right)$. When this condition is satisfied, selection favors a pure CLC strategy as soon as the rate of predation $(p)$ exceeds the threshold value $p^{*}$, defined by equation (10). Examining equation (10), we can see that the greater the ratio $\mu_{3} / \mu_{2}$ and the difference $N_{2}-N_{1}$, the lower the value of $p^{*}$. If the intermediate-host density is higher than the definitive-host density $\left(N_{2}>N_{1}\right)$, should 
$p$ exceed $p^{*}$, then selection will favor a pure CLC strategy. Once a pure CLC is retained by selection, further increase of the rate of predation $p$ through intermediate-host manipulation may occur, which may, in return, reinforce the selective advantage of the CLC.

\section{Generalization to the Full Model}

We explored the influence of the trade-off form on our model and looked at what happens for different trade-off relationships between an SLC and a CLC over 10 different $x$ values between 0 and 10 . The qualitative shapes of the graphs are the same for all highly costly trade-offs between an SLC and a CLC strategy ( $x$ values below 1). The same is true for the less costly trade-offs ( $x$ values above 1). Figure 4 shows examples of one more costly trade-off (left column) and one less costly trade-off (right column). The central column $(x=1)$ illustrates again the four graphs of figure 3. Comparing the columns of figure 4 , we can see that a more costly trade-off between an SLC and a CLC strategy makes the SLC less invasible by the CLC and that a less costly trade-off between an SLC and a CLC strategy makes the SLC more easily invasible by the CLC. In the case of a highly costly trade-off between an SLC and a CLC (first column of fig. 4), we still do not see any adaptive mixed strategy between the pure SLC and CLC strategies, but we do now have the two pure strategies (SLC and CLC) as adaptive peaks. Thus, in this context, whether selection favors an SLC or a CLC depends on the initial value of the definitive-host infection rate $\beta_{1}$. However, because the initial state in our model is a pure SLC $\left(\beta_{1}=A\right)$, the conclusion is that evolution always favors a pure SLC strategy except if other evolutionary forces decrease $\beta_{1}$. Examining equation (3), we can see that what really counts in the expression of $R_{0}$ is the product of $\beta_{1}$ and $N_{1}$, more than either of these two parameters alone. Thus, decreasing $N_{1}$ relative to $N_{2}$ should have the effect of increasing the value of $\beta_{1}$ below which selection will favor a CLC. Figure 5 verifies this prediction, where for high values of predation rate $p$ we reach the extreme case where the minimum of the curve coincides with $\beta_{1}=A$. Thus, for high prey density (high $N_{2} / N_{1}$ ratio; fig. 5), we have the same conclusion for $x<1$ as for $x=1$; the adaptive strategy switches from SLC to CLC as $p$ increases. In the case of a less costly trade-off between an SLC and a CLC strategy (third column of fig. 4), we can see the emergence of an evolutionary stable mixed strategy $\left(R_{0}\right.$ maximized for intermediate levels of $\beta_{1}$ ) between the pure SLC and pure CLC strategies. The ratio $N_{2} / N_{1}$ now has little effect on the qualitative shape of the curves for $x>$ 1 (fig. 5), which is not surprising. Indeed, we expect the relative host densities to have more influence on the results when the exploitation of one life cycle strongly penalizes the adoption of the other (more costly trade-off) than when the two life cycles can coexist to a certain degree (less costly trade-off). Selection favors a higher proportion of the CLC in a mixed strategy when predation rates are high. Once a mixed strategy has emerged, selection could then favor increases in $p$ via host manipulation and render them more susceptible to predation. If this happens, the consequence of host manipulation would be an increase in the benefits linked to the CLC in the mixed strategy and possibly the selection of a pure CLC.

\section{Discussion}

Our simple model shows that adding an intermediate-prey host in the cycle of trophically transmitted parasites is advantageous under certain conditions. The necessary condition is that the intermediate-host density must be higher than the definitive-host density. Given this condition, factors that favor the switch from SLC to CLC are a high predation rate and a low mortality rate in the intermediate host compared with the mortality rate in the environment.

We assumed that the parameters illustrated in figure 1 are independent. This may not be the case because tradeoffs may exist between certain parameters. For example, there might be a trade-off between how many parasites are shed from an infected host and the life span of this host (Bonhoeffer et al. 1996) or a trade-off between the longevity of the parasite in the host and in the environment (Ebert 1998; Regoes et al. 2000). Nonindependence of the parameters may limit the parameter space and constrain possible transitions between SLCs and CLCs in specific cases. Note that fecundity, while often considered an important element in many trade-offs (Stearns 1992), does not play a role in determining transitions between CLCs and SLCs (see eq. [3]).

Parasites are faced with a highly heterogeneous environment because their hosts are patchily distributed in space and host densities may vary considerably over time. Parasites must, therefore, seek ways of locating and exploiting this highly dispersed resource. Trophic transmission might have evolved in response to these transmission constraints because, similarly to what occurs in numerous free-living plants and animals, the use of a carrier host can considerably improve the probability of reaching a suitable habitat. However, the need for parasites to adjust to historical contingencies might have constrained opportunities for the selection of CLCs (Smith Trail 1980). Unfortunately, the lack of information on most parasite life histories limits our ability to provide examples from nature that mirror our models. In many cases, a nematode infecting an inappropriate host may wander in the host tissues (the nematode eventually dies, and this host suffers 
some pathology). If the nematode's correct host were to eat this inappropriate host, the life cycle might have a chance of completion, which would provide an opportunity for the evolution of a CLC. Another particular example that could be mentioned here is the case of coprophagy. If parasite eggs are mostly being eaten by scavengers, they may have a strong selection pressure to survive in the scavenger until the scavenger is eaten by the correct host. For example, Baylissascaris procyonis is a nematode that infects raccoon guts. Rodents scavenge raccoon feces, get infected with wandering larvae, become sick, and are then captured by raccoons. Raccoons can also be infected directly through coprophagy.

Assuming that the addition of hosts to SLCs has evolved to improve the efficiency of transmission, we find that several predictions arise. Since we assume that CLCs involving trophic transmission are adaptations to increase transmission, CLCs should mainly evolve when the target host (definitive host) is difficult to reach by self-dispersing propagules. Conversely, all things being equal, parasites exploiting a host species easy to infect by self-dispersing propagules should more often have SLCs than CLCs. It remains unclear, however, what makes a host "difficult" or "easy" to infect by self-dispersing propagules since, undoubtedly, numerous factors related to both the host (e.g., size, abundance, behavior, etc.) and the parasite (e.g., size, sensory apparatus, life expectancy, etc.) are involved. In this context, mechanisms of infection that shape the tradeoffs between SLC and CLC evolution are also important to consider. Indeed, if definitive and intermediate hosts are infected using similar mechanisms, then the trade-off is likely to be less costly. It remains difficult to illustrate these predictions concretely from real examples because, for most CLC parasites, the ancestral host is not adequately known (Poulin 1998). Because this information is essential to understand the selective factors that have favored the addition of hosts to life cycles (see the introduction to this article), testing the current hypotheses on the evolution of CLCs remains difficult. Mixed strategies, such as the facultative intermediate host used by the raccoon nematode, may provide good systems to investigate. Also, cercariae of the fluke Fasciola hepatica can be transmitted to vertebrate hosts by encysting on leaves of water plants (i.e., use of an intermediate host) but also by producing floating metacercariae (i.e., self-dispersal; Vareille-Morel et al. 1993; Mas-Coma and Bargues 1997). Although we do not know how such a polymorphism in transmission is maintained, this example illustrates the strategic choices that confront parasites. The observation of only very few mixed evolutionary stable strategies between SLCs and CLCs in nature suggests that trade-offs are normally substantial; this constitutes a subject of further investigation.

Although our hypothesis does not attempt to explain the evolution of all CLCs involving trophic transmission, we believe that the scenario presented here deserves consideration because it is a simple mechanism able to explain the transition from an SLC to a CLC when the ancestral host is a predator. Our assumption that contact with prey does not result in the death of the larva of an SLC parasite could be violated in many systems. This is particularly true for situations where a prey item might ingest and digest eggs or provide a target for unsuccessful penetration by free-living larvae. In this case, we will have underestimated mortality for the SLC strategy and, thus, have increased the selective advantage of a CLC. From a theoretical point of view, we cannot exclude that selection may eventually favor the subsequent elimination of the predator in the CLC and lead to a new SLC exploiting the prey. Indeed, if the prey are common and good hosts, there will be some conditions under which selection favors host switching, not evolution of a CLC. Further information on the evolutionary history within groups of CLC parasites would be necessary to assess the relevance of these hypotheses to explain the evolution of complexity in parasitic life cycles.

\section{Acknowledgments}

We thank R. Hechinger and K. Phillips for comments on an earlier draft of the article. We are also grateful to $\mathrm{H}$. McCallum and two anonymous referees for the considerable improvement they brought to the first version of the manuscript. M.C. is supported by a Bourse de DocteurIngénieur du Centre National de la Recherche ScientifiqueRégion Languedoc-Roussillon. S.P.B. is supported by the European Marie Curie Fellowship Programme. F.T. is supported by an Action Concertée Incitative jeune chercheur from the Centre National de la Recherche Scientifique.

\section{Literature Cited}

Anderson, R. C. 1992. Nematode parasites of vertebrates: their development and transmission. CAB International, Cambridge.

Anderson, R. M., and R. M. May. 1982. Coevolution of hosts and parasites. Parasitology 85:411-426.

Bonhoeffer, S., D. Ebert, and R. E. Lenski. 1996. The curse of the pharaoh: the evolution of virulence in pathogens with long living propagules. Proceedings of the Royal Society of London B, Biological Sciences 263:715-721.

Brown, S. P., F. Renaud., J. F. Guégan, and F. Thomas. 2001. Evolution of trophic transmission in parasites: the need to reach a mating place? Journal of Evolutionary Biology 14:815-820.

Chabaud, A. G. 1954. Sur le cycle évolutif des spirurides et des nématodes ayant une biologie comparable: valeur systématique des caractères biologiques. Annales de Parasitologie Humaine et Comparée 29:42-88. 
Combes, C. 1991a. Ethological aspects of parasite transmission. American Naturalist 138:866-880.

- 1991b. Evolution of parasite life cycles. Pages 62-82 in C. A. Toft, A. E. Aeschlimann, and L. Bolis, eds. Parasite-host associations: coexistence or conflict? Oxford University Press, Oxford.

- 1995. Interactions durables: ecologie et evolution du parasitisme. Masson, Paris.

Combes, C., A. Fournier, H. Moné, and A. Théron. 1994. Behaviours in trematode cercariae that enhance parasite transmission: patterns and processes. Parasitology 109: S3-S13.

Dobson, A. P. 1989. The population biology of parasitic helminths in animal populations. Pages 145-175 in S. A. Levin, T. G. Hallam, and L. J. Gross, eds. Applied mathematical ecology. Springer, London.

Dobson, A. P., and A. Merenlender. 1991. Coevolution of macroparasites and their hosts. Pages 83-101 in C. A. Toft, A. E. Aeschlimann, and L. Bolis, eds. Parasite-host associations: coexistence or conflict? Oxford University Press, Oxford.

Ebert, D. 1998. Experimental evolution of parasites. Science (Washington, D.C.) 282:1432-1435.

Esch, G. W., and J. C. Fernandez. 1993. A functional biology of parasitism: ecological and evolutionary implications. Chapman \& Hall, London.

Ewald, P. W. 1995. The evolution of virulence: a unifying link between parasitology and ecology. Journal of Parasitology 81:659-669.

Gibson, D. I., and R. A. Bray. 1994. The evolutionary expansion and host-parasite relationships of the Digenea. International Journal of Parasitology 24:1213-1226.

Lafferty, K. D. 1992. Foraging on prey that are modified by parasites. American Naturalist 140:854-867.

. 1999. The evolution of trophic transmission. Parasitology Today 15:111-115.

Lafferty, K. D., and A. K. Morris. 1996. Altered behavior of parasitized killifish increases susceptibility to predation by bird final hosts. Ecology 77:390-1397.

Mackinnon, M. J., and A. F. Read. 1999. Genetic rela- tionships between parasite virulence and transmission in the rodent malaria Plasmodium chabaudi. Evolution 53:689-703.

Mas-Coma, S., and M. D. Bargues. 1997. Human flukes: a review. Research and Reviews in Parasitology 57: 145-218.

May, R. M., and M. A. Nowak. 1994. Superinfection, metapopulation dynamics and the evolution of diversity. Journal of Theoretical Biology 170:95-114.

Morand, S. 1996. Biodiversity of parasites in relation to their life cycles. Pages 243-260 in M. E. Hochberg, J. Clobert, and R. Barbault, eds. Aspects of the genesis and maintenance of biological diversity. Oxford University Press, Oxford.

Nowak, M. A., and R. M. May. 1994. Superinfection and the evolution of parasite virulence. Proceedings of the Royal Society of London B, Biological Sciences 255: 81-89.

Poulin, R. 1998. Evolutionary ecology of parasites: from individuals to communities. Chapman \& Hall, London.

Regoes, R. R., S. Bonhoeffer, and M. A. Nowak. 2000. Evolution of virulence in a heterogeneous host population. Evolution 54:64-71.

Rohde, K. 1994. The origins of parasitism in the Platyhelminthes. International Journal of Parasitology 24: 1099-1115.

Smith Trail, D. R. 1980. Behavioral interactions between parasites and hosts: host suicide and evolution of complex life cycles. American Naturalist 116:77-91.

Stearns, S. C. 1992. The evolution of life histories. Oxford University Press, Oxford.

Thomas, F., K. Mete, S. Helluy, F. Santalla, O. Verneau, T. de Meeüs, F. Cézilly, and F. Renaud. 1997. Hitch-hiker parasites or how to benefit from the strategy of another parasite. Evolution 51:1316-1318.

Vareille-Morel, V., G. Dreyfuss, and D. Rondelaud. 1993. Premières données sur la dispersion et le devenir des métacercaires flottantes de Fasciola hepatica L. Bulletin de la Société Française de Parasitologie 11:63-69.

Associate Editor: Curt Lively 